\title{
Collisions, Cosmic Radiation and the Colors of the Trojan Asteroids
}

\author{
M.D. Melita ${ }^{1}$ \\ Instituto de Astronomía y Física del Espacio. Buenos Aires. Argentina. \\ melita@iafe.uba.ar \\ and \\ G. Strazzulla ${ }^{2}$ \\ INAF-Osservatorio Astrofisico di Catania, Italy \\ gianni@oact.inaf.it \\ and \\ A. Bar-Nun ${ }^{3}$ \\ Department of Geophysics and Planetary Sciences.Tel Aviv University,Tel Aviv,Israel. \\ akivab@post.tau.ac.il
}

Received —

Submitted to ICARUS 


\begin{abstract}
The Trojan asteroids orbit about the Lagrangian points of Jupiter and the residence times about their present location are very long for most of them. If these bodies originated in the outer Solar System, they should be mainly composed of water ice, but, in contrast with comets, all the volatiles close to the surface would have been lost long ago. Irrespective of the rotation period, and hence the surface temperature and ice sublimation rate, a dust layer exists always on the surface. We show that the timescale for resurfacing the entire surface of the Trojan asteroids is similar to that of the flattening of the red spectrum of the new dust by solar-proton irradiation. This, if the cut-off radius of the size distribution of the impacting objects is between $1 \mathrm{~mm}$ and $1 \mathrm{~m}$ and its slope is -3 , for the entire size-range. Therefore, the surfaces of most Trojan asteroids should be composed mainly of unirradiated dust.
\end{abstract}

Subject headings: Trojan Asteroids. Asteroids, Surfaces. Comets. Solar System, Origin. 


\section{Introduction}

All the Trojan asteroids orbit about the Sun at roughly the same heliocentric distance as Jupiter, residing close to the Lagrangian stable points, leading or trailing at $\sim 60^{\circ}$ from the planet. They are particularly interesting because of being relatively isolated, lying just beyond the snow line, the distance at which water can exist in the form of ice. As such, it may be expected that the bulk of the Trojans were mainly composed of ices. However, at the moment, no water or other more volatile substances have been detected on the surface of a Trojan (Yang \& Jewitt 2007, Emery \& Brown 2003; 2004). In the case of Ennomos, an unusually hight-albedo object, the surface-content of water ice has been quantified to be below 10\% in mass (Yang \& Jewitt 2007). However the lack of detection does not imply that water ice is not there. Brunetto and Roush (2008) have in fact shown that a relatively thin (tens of microns) crust of specific refractory materials (they considered irradiated methane ice) can mask the presence of water ice bands in the spectrum.

The Trojans have been characterized as similar to cometary nuclei or extinct comets (Jewitt and Luu 1990). Most of the objects fall into the D and P asteroid taxonomic classes and a few objects show spectra with neutral or even negative slopes in the visual range (Bendoya et al. 2004), with the majority having moderately-red surfaces (see figure 1). They have been observed to posses low albedos (Fernandez et al. 2003) of about $4 \%$ and infrared observations indicate the presence of silicates (Cruikshank et al. 2001, Emery et al. 2006) and depletion of water ice and volatiles (Emery \& Brown 2003; 2004).

Numerical models of the collisional evolution of the Trojan asteroids (Marzari et al. 1997) are successful in explaining the present Trojan population (Jewitt et al. 2000). The steep slope of the Trojan size distribution at diameters larger than about $50-100 \mathrm{~km}$ is essentially unaltered during the collisional evolution and must reflect the formation process of these bodies. At smaller sizes, collisions have produced a power law size distribution, 
with a slope characteristic of collisionally relaxed populations. The formation of prominent dynamical families in the Trojan swarms is a natural outcome of the collisional process, and indeed a significant number of families are known (see for example Beauge \& Roig 2001).

It has also been found that the absolute-magnitude distribution of the Trojans in stable orbits is bimodal, while that of the unstable orbits is unimodal, with a slope similar to that of the small stable Trojans (Melita et al. 2008a). This supports the hypothesis that the unstable objects are mainly byproducts of physical collisions, the transitional size has been found to be $H \approx 9.5$. But the slopes of the reflectance spectra show no relation with the size of the objects (see figure 2) or with the stability of the orbits (Melita et al. 2008a).

We intend to understand why most of the Trojans are moderately red, regardless if they are large bodies or fragments of collisions. These asteroids are subject to impacts which can extract fresh material from the interior and scatter it on the surface. The fresh material would be composed of dust and ice. After the Deep-Impact event, we note the increase of emission of both organic compounds and water ice (Lisse et al. 2006). But, if water ice were deposited on the surface after an impact, it would sublimate very rapidly. Therefore, after a physical impact, a layer of unirradiated ice -which has a typically red spectral slope- is deposited in the surface. It has been suggested that terrestrial bitumens are good spectral analogues for such a dust layer (e.g. Moroz et al 2004 and references therein). The less energetic solar protons modifies the spectroscopic properties of this dust, reducing its albedo and flattening the spectral slope (Moroz et al. 2004). But if the collisional process can resurface the asteroids on a timescale which is similar, or shorter, than the timescale that flattens the spectrum, it is expected that most of the Trojans will be observed to be red. In the following section we present a model to estimate relevant timescales and we discuss its results in the last section. 


\section{Surface alteration timescales}

\subsection{Timescale of flattening of the spectral slope by irradiation}

There are a number of energetic ion populations bombarding the surfaces of small bodies in the outer Solar System: solar wind ions, ions from solar flares, galactic cosmic rays, and the so called "anomalous" cosmic ray component. The last component is however relevant only to objects at the Pluto orbit and beyond.

Solar wind is an expanding flux of fully ionized plasma that reaches, at distances greater than a few solar radii, an expansion speed of about $400 \mathrm{~km} \mathrm{~s}^{-1}$ corresponding to an energy of $\approx 1 \mathrm{keV} / \mathrm{amu}$ (slow solar wind, see e.g. Gosling 2007). At 1 AU its flux is $\approx 10^{8}$ protons $\mathrm{cm}^{-2} s^{-1}$ and it decreases with the square of the solar distance. The effects of the solar wind ions irradiating "red" organic bitumens (namely asphaltite and kerite) have been simulated in the laboratory (Moroz et al 2004). The hypothesis is that terrestrial bitumens are good spectral analogues of the red organic surfaces of small objects in the Solar System (we consider Trojans in the present paper). The main result is a progressive flattening of the red spectrum of bitumens with ion fluence. Moreover Moroz et al (2004) suggest that the mechanism that produces the flattening is connected to the energy deposited by elastic collision of the incoming ions with the atoms in the target. This allows to extend the laboratory results obtained with 10 's $\mathrm{keV} \mathrm{H}^{+}, \mathrm{N}^{+}$, and $\mathrm{Ar}^{++}$to all solar wind ions. Because in the laboratory the flattening of the asphaltite spectrum in the $0.3-0.8$ micrometers spectral region has been obtained after about $0.4 \times 10^{18} \mathrm{C}$-displacements $\mathrm{cm}^{-2}$ (see Moroz et al, top panel in Fig 14), we need to calculate the time necessary to solar wind ions (at $5 \mathrm{AU})$ to cause the accumulation of such an amount of damage on the bombarded surface. As said, solar wind ions have energy of about $1 \mathrm{keV} / \mathrm{amu}$. Most of them are protons (96\%) and He $(4 \%)$ with traces of heavier elements. Using the TRIM code (Transport of Ions in Matter; e.g., Ziegler et al. 1996) we calculate that the number of displaced atoms in 
asphaltite is about 1.5 for $1 \mathrm{keV}$ protons and 20 for $4 \mathrm{keV}$ alpha particles. Considering the respective abundances, we obtain an average of 2.2 C-displacements per solar wind ion (the contribution of heavier ions can be neglected). At a distance of 5 AU from the Sun we then expect that an exposed surface will suffer about $10^{8} \times 2.2 / 25=9 \times 10^{6} \mathrm{C}$-displacements $\mathrm{cm}^{-2} \mathrm{sec}^{-1}$. Thus the time necessary to reproduce the effects observed in the laboratory is: $0.4 \times 10^{18} / 9 \times 10^{6}=5.5 \times 10^{10}$ sec i.e. about $2 \times 10^{3}$ yrs.

It is important to note that solar wind particle irradiation affects only a thin surface layer $(\leq 2000 \AA$ ) . This is however sufficient to affect the optical properties of the surface (as simulated in the laboratory), but may be easily destroyed by impact resurfacing. As discussed here, such resurfacing of irradiated material probably occurs constantly.

In order to produce a radiation-induced damage of deeper layers, more energetic ions should be considered. Solar cosmic rays are produced during solar flares. An average flux of solar protons having an energy of $100 \mathrm{keV}$ and a penetration depth of about $1 \mu \mathrm{m}$ has been estimated to be $\approx 2 \times 10^{11}$ protons $\mathrm{cm}^{-2} \mathrm{yrs}^{-1}$ (Lanzerotti et al., 1978). It is inversely proportional both to the square of the energy and to the square of the solar distance. At 5 $\mathrm{AU}$ the time necessary to reach the maximum C-displacements of our experiments is about $2 \times 10^{7}$ yrs.

Galactic cosmic rays are less abundant but significantly more penetrating. In a recent review Hudson et al. (2008, and references therein) reported the estimate of the radiation doses for objects at different distances from the Sun (see their Table 1). It has been found that objects orbiting at 5-35 AU accumulate in about $10^{9}$ yrs an irradiation damage sufficient to be fully chemically altered down to depth of the order of 1 meter. Although galactic energetic particles transfer most of their energy through electronic loss (ionizations and excitations), however at the end of their path they slow down and are thought to produce, in the so called Bragg peak, the same effects meassured in the laboratory for low 
energy particles.

In summary we have shown that the uppermost layers of an object orbiting at the Jupiter distance can be weathered in about $10^{3}$ yrs. If its surface is an organic material spectrally similar to bitumens, its red sloped spectrum is flattened on that time scale. Deeper layers are weathered in longer times: at a depths of the order of 1 meter the target is fully altered in about $10^{9}$ yrs.

\subsection{Timescale of sublimation of amorphous water-ice}

The timescale for sublimation-mantle growth, $\tau_{M}$, is (Jewitt 2002):

$$
\tau_{M}=\frac{\rho L}{f_{M} d m / d t}
$$

where $L$ is the depth of the sublimated material, $\rho$ is the mass density of the asteroid, $f_{M}$ is the fraction of the solid mass that cannot be ejected by gas drag and $d m / d t$ is the drag mass-loss rate per unit area.

For a fast rotator, the effective temperature at the surface of a Trojan is $\sim 120^{\circ} \mathrm{K}$ -assuming an albedo of 0.04. At this temperature, the mass loss rate of perfectly absorbing

water-ice in equilibrium sublimation is approximately $d m / d t \approx 310^{-11} \mathrm{~kg} \mathrm{~m}^{-2} \mathrm{~s}^{-1}$. To find a reliable estimation for $d m / d t$ at $T=120^{\circ} \mathrm{K}$ is quite a complicated problem and we have reached this value after much deliberation, following the comments of the reviewer. The water-vapor pressure in torr, $P$, vs. temperature, $T$, was calculated, using the polynomial from the International Critical Tables between $140^{\circ} \mathrm{K}$ and $180^{\circ} \mathrm{K}$ :

$$
\log (P)=\frac{-2445.5646}{T}+8.23121 \times \log (T)-0.167706 \times T+1.20514 \times 10^{-5} T^{2}-6.757169,
$$

and checked against the $P$ vs. $T$ in the CRC-Handbook of Chemistry and Physics and found to agree with Sack and Baragiola (1993). The pressure at $120^{\circ} \mathrm{K}$ is $1.8 \times 10^{-10} \mathrm{~Pa}$. 
Since the flux of sublimating water molecules, $F$, is,

$$
F=\frac{P(T)}{(2 \pi K m T)^{1 / 2}},
$$

where $K$ is Boltzmann's constant and $m$ the mass of the molecule. We recalculated the square root term in the denominator and found it to be: $(2 \pi K m T)^{1 / 2}=1.77 \times 10^{-23} \mathrm{~kg} \mathrm{~m}^{-1} \mathrm{~s}^{-1}$ per molecule. Hence the flux calculated from the pressure as obtained by the polynomial is: $F\left(T=120^{\circ} K\right)=\frac{P\left(T=120^{\circ} K\right)}{(2 \pi K m T)^{1 / 2}}=1.1 \times 10^{13}$ molec. $m^{-2} s^{-1}=1.1 \times 10^{9}$ molec. $\mathrm{cm}^{-2} \mathrm{~s}^{-1}$. This flux was compared with the flux meassured by Ntesco and Bar-Nun (2005) in their Figure 1 and we realized that the measured value at $120^{\circ} \mathrm{K}$ is the background of water vapor in our vacuum chamber. Extrapolating the flux vs T plot in their Figure 1 to $120^{\circ} \mathrm{K}$ leads to a flux of approximately $10^{11}$ molec. $\mathrm{cm}^{-2} \mathrm{~s}^{-1}$, which is 100 times larger than the one calculated by the $\mathrm{P}$ vs $\mathrm{T}$ through the polynomial, due to the very large surface area of the amorphous ice, much larger than the area calculated by the geometric dimension of the substrate on which the ice was deposited. As measured by Bar-Nun et al. (1987), by Ar adsorption at $120^{\circ} \mathrm{K}$, a surface area of $38 \mathrm{~m}^{2} \mathrm{~g}^{-1}$ was found, in good agreement with Mayer and Pletzer (1986) who measured $40 \mathrm{~m}^{2} \mathrm{~g}^{-1}$ at $114^{\circ} \mathrm{K}$. Sack and Baragiola' s (1993) Figure 2 data can be extrapolated to $8 \times 10^{10}$ molec. $\mathrm{cm}^{-2} \mathrm{~s}^{-1}$ at $120^{\circ} \mathrm{K}$ and shows an 8 fold increase in the vapor pressure in amorphous non annealed ice, which they attribute to the larger surface area. These authors (Figure 3)show a 7 fold increase in the flux at $135^{\circ} \mathrm{K}$ with an ice thickness between 0.12 and $5.1 \mu \mathrm{m}$ and a $7-8$ fold decrease at $135^{\circ} \mathrm{K}$ during 200 min at that temperature. Both observations point to a considerable effect in surface roughness. To be on the conservative side we shall adopt a flux of $510^{10}$ molec. $\mathrm{cm}^{-2} \mathrm{~s}^{-1}$ or $1.510^{-11} \mathrm{~kg} \mathrm{~m}^{-2} \mathrm{~s}^{-1}$. It should be remembered that slight deviations from an albedo of 0.4 or the fast rotator approximation could result in large changes in the rate of sublimation. The question is then which flux of water molecules from an ice surface at $120^{\circ} \mathrm{K}$ should be adopted?. The one obtained from Notesco and Bar-Nun's (2005), $10^{11}$ molec. $\mathrm{cm}^{-2} \mathrm{~s}^{-1}$ 
or Sack and Baragiola's (1993) extrapolated $8 \times 10^{10}$ molec. $\mathrm{cm}^{-2} \mathrm{~s}^{-1}$ ?. Since this mass loss rate is very low, even the smallest sub-micron sized grains on the surface of the Trojan asteroids are retained, hence, for the fast rotator case, we take $f_{M}=1$.

The known rotation periods of Trojans range approximately from $4 h r$ to $40 h r$ (Hartmann et al. 1988, Binzel and Sauter 1992, Melita et al. 2008b)1.

As one extreme case, in the fast rotator approximation, the time that it takes to sublimate a layer equal to the penetration-depth of the solar protons, roughly a $100 \mu m$ dust layer, is only of $\sim 200 \mathrm{yr}$.

In the case of the slower rotators we may take, as another extreme case, the stationary approximation. The stationary Trojan temperature of the side facing the Sun can be estimated using the equation of effective temperature with back irradiation from half a sphere, rather than the entire surface of the sphere as in the case of a fast rotator. This leads to a higher temperature, namely $T_{S} \approx 168^{\circ} \mathrm{K}$, and the corresponding mass-loss rate $d m / d t\left(T_{S}\right) \approx 310^{-4} \mathrm{~kg} \mathrm{~m}^{-2} \mathrm{~s}^{-1}$ (CRC-Handbook of Chemistry and Physics 1981-1982). In this case, the fraction of the solid mass that cannot be ejected by gas drag can be estimated as (Jewitt 2002):

$$
f_{M}=\frac{\log \left(a_{M} / a_{c}\right)}{\log \left(a_{M} / a_{m}\right)}
$$

where $a_{M}$ and $a_{m}$ are the maximum and the minimum grain sizes in the distribution respectively and $a_{c}$, the maximum particle-radius that is retained, given by:

$$
a_{c}=\frac{v_{e}(R) d m / d t\left(T_{S}\right)}{G R \rho^{2}},
$$

where $v_{e}(R)$ is the escape velocity from a body of size $R$ and $G$ is the gravitational constant. If we take $a_{M}=1 \mathrm{~mm}$ and $a_{m}=1 \mu \mathrm{m}$ and $R=100 \mathrm{~km}$, then $a_{c} \approx 2 \mu \mathrm{m}$. We find that, for a

\footnotetext{
${ }^{1}$ Rotation periods of Jupiter Trojan asteroids, determined before March 2006, can be found at the Minor Planet Center website, http://cfa-www.harvard.edu/cfa/ps/mpc.html
} 
body of rotational period $P=40 h r$, the timescale in which an insulator-mantle of thickness $L=100 \mu m$ grows, is only $\tau_{M}(P=40 h r) \approx 3$ days.

\subsection{Timescale of collisional re-surfacing}

To compute the timescale of the collisional resurfacing process we have adapted the model by Gil-Hutton (2002) and incorporated the latest knowledge gained by the Deep Impact mission (see for example Richardson et al. 2007).

The time to cover the entire surface of an asteroid of radius $R$ with debris extracted from below the surface by the action of physical collisions, $\tau_{C R}$, is given by:

$$
\tau_{C R}=\frac{1}{\dot{S}_{r}}
$$

where $\dot{S}_{r}=\dot{S} /\left(4 \pi R^{2}\right)$ is the fraction of the surface that is covered by material excavated by collisions per year and $\dot{S}$ is the total area covered by collisional ejecta each year.

The total area covered by ejecta per year, $\dot{S}$, for an object of radius $R$ is:

$$
\dot{S}=\int_{r_{\min }}^{r_{\max }} \dot{N}(r) A_{E}(r) d r
$$

where $\dot{N}(r)$ is the number of collisions per unit time and $A_{E}(r)$ is the area covered by the ejecta produced when projectiles of radii $r$ collide with an object of radius $R$, at a typical encounter velocity $v$. We write $A_{E}$ as $A_{E}=l \times A_{c}(R, r, v)$, where $A_{c}(R, r, v)$ is the area of the crater produced and $A_{c}=\pi d^{2}$, where $d$ is the diameter of an idealized crater, and we assume the densities of the target and the impactor to be equal, i.e. $\rho_{R}=\rho_{r}=1.5 \mathrm{gcm}^{-3}$. The volume of the crater in the gravity dominated cratering regime is estimated as (Richardson et al. 2007, Holsapple 1993):

$$
V_{g}=K_{1}\left(\frac{m}{\rho}\right)\left(\frac{r g(R)}{v^{2}}\right)^{\frac{-3 \mu}{2+\mu}}
$$


where $g(R)$ is the surface gravity of the target, $m$ is the mass of the impactor, $v$ its impact velocity, $\rho$ is the density which is assumed to be equal for the target and the impactor. The scaling constants $K_{1}=0.24$ and $\mu=41$, correspond to a loosely bound material such as sand, which gave a good agreement with the size of the crater produced in the Deep impact mission (Richardson et al. 2007). The diameter of the crater is easily obtained from

$$
V_{g}=\frac{1}{24} \pi d^{3}
$$

Now, we can write $\dot{N}$ as,

$$
\dot{N}=P_{i}(R+r)^{2} d N(r)
$$

where $P_{i}$ is the intrinsic probability of collisions, as defined by Wetherill (1967), is the probability of collision per unit time, per unit cross section and per number of colliding pairs and $d N(r)$ is the number of objects with radii $r$. The values of $P_{i}$ and $v$, have been computed specifically for the Trojans by Dell'Oro et al. (1998) giving approximately $6.510^{-18} \mathrm{~km}^{-2} \mathrm{yrs}^{-1}$ and $5 \mathrm{~km} \mathrm{~s}^{-1}$ respectively.

The integral on equation 1 depends critically on the limits, which must be handled carefully. The upper limit, $r_{\max }$, is the maximum radii of the projectiles that do not shatter a target of radius $R$. For the $\rho_{R}=\rho_{r}$ case is,

$$
r_{\max }=R\left(5 \beta v_{c}^{2}-1\right)^{-1 / 3}
$$

(Petit \& Farinella 1993), where $\beta=10^{-8} \mathrm{~g} \mathrm{erg}^{-1}$ is a crater excavation coefficient. While $r_{\min }$ is the cut-off radius.

Finally, to compute $\tau_{C}$, we need an estimation of the observed distribution of sizes in the Trojan population, which, for the smallest objects is (Jewitt et al. 2000),

$$
d N(r)=1.510^{6}\left(\frac{1 k m}{r}\right)^{3} .
$$




\subsubsection{The choice of $r_{\text {min }}$, the cut-off radius}

The contribution of the dust phase, i.e. $\mu m$ sized particles, to the modification of the a $100 \mu \mathrm{m}$ layer of irradiated material is negligible, since it would only produce re-arrangements. For such small impactors, the cratering regime is strength-dominated. The volume of the crater produced $V_{S}$ can be estimated as (Richardson et al 2007, Holsapple 1993):

$$
V_{S}=K_{1} \frac{m}{\rho}\left(\frac{\bar{Y}}{\rho v^{2}}\right)^{-\frac{3}{2} \mu},
$$

where $\bar{Y}$ is the effective yield strengths of the target material. We adopt a value of $\bar{Y} \approx 10 P a$, as determined for comet Temple 1 and also in agreement with laboratory experiments (Richardson et al. 2007, Bar-Nun et al. 2007). Under the assumptions used previously, the depth of this crater for an impactor of size $10 \mu m$ is $600 \mu m$, i.e., roughly the penetration depth of the solar protons.

Moreover, micro-impacts contribute less to the resurfacing because dust is carried away by radiation and its size distribution is much shallower than the one corresponding to macroscopic objects. Therefore the smallest cut-off radius that we shall assume is $1 \mathrm{~mm}$, since objects of this size are not affected by radiation. Unfortunately, there is no information available on the size distribution of objects of sizes ranging between $1 \mathrm{~mm}$ and $100^{\prime} \mathrm{s} \mathrm{m}$ orbiting in the Trojan clouds. We assume that the size distribution of the smallest observed Trojans (Jewitt et al. 2000) also applies for small particles. Although this assumption may seem extreme, there is no creation or destruction mechanism that affects the small particles differently than the large objects, since none of them are affected by radiation. And the evidence of the cratering record on asteroids indicates that the slope of the size distribution of impactors is unaltered from $\mathrm{km}$-sized bodies down to $\mathrm{m}$-sized objects (O'Brien et al. 2006).

If we take $l=1$, the timescale of collisional resurfacing is, neglecting overlapping, the 
time-span in which all the surface is covered with craters, $\tau_{C}$. Note that $\tau_{C}$ is larger than the time to cover the object with ejecta. Values of $\tau_{C}$ for the size range of the known Trojan asteroids, are shown in figure 3 for different values of the cut-off radius $r_{\text {min }}$. For a value of $r_{\min }=1 \mathrm{~mm}$ the object is completely covered by craters in about 700 to $1100 \mathrm{yr}$, while for $r_{\min }=10 \mathrm{~m}$, it occurs in $10^{4}$ to $10^{5} \mathrm{yrs}$.

Now we estimate how the area covered by optically thick ejecta, scales with the size of the crater. If we assume that the optically thick ejecta is close to the edge of the crater, and is mostly originated by material close to it, then the ejection velocity from the impact, $v_{e}$, scales as (Richardson et al. 2007):

$$
v_{e} \propto \sqrt{g \frac{d}{2}} .
$$

Then, for the distance travelled by the ejecta, $h$, is:

$$
h \propto \frac{v_{e}^{2}}{g}=\frac{d}{2},
$$

and therefore, the ratio

$$
l=\left(\frac{h}{d / 2}\right)^{2}
$$

is constant. To fix this constant we use the fact that the size of the crater produced in comet Temple 1 by the Deep Impact mission has a radius of approximately $30 \mathrm{~m}$ and the base of the plume after the event had a radius of about 150m (Richardson et al. 2007), hence we adopt a value for the ratio of the areas of $l=25$. In figure 3 we also plot values of the timescale for collisional resurfacing, $\tau$, assuming a value of $l=25$. For a value of $r_{\min }=1 \mathrm{~mm}$ the resurfacing occurs in about 100 to $300 \mathrm{yr}$, while for $r_{\min }=10 \mathrm{~m}$, it occurs in 3000 to $7000 y r s$. 


\section{Conclusion}

The surface properties of a Trojan asteroid are determined by the interplay of three different mechanisms. When an impact occurs, if it is sufficiently energetic, the inner materials of the body are exposed and scattered on the surface. Given that the site of residence and formation of the Trojans is the outer Solar System, water ice could be abundant, but, as shown in section 2.2, it sublimates fast from the surface, leaving a mantle of dust, most probably with a red spectroscopic slope. If this dust layer would remain unaltered for more than $10^{3} \mathrm{yrs}$, its spectroscopic slope would turn to neutral by the action of solar protons. But impacts occur so frequently that the irradiation mantle is disrupted.

The surfaces of most Trojans are red, but some neutral objects exists, therefore, it is suggested that the irradiation resurfacing timescale is similar, but not much shorter, than the collisional one. The conclusion reached here is that the timescale to fill the entire surface of the Trojan asteroids with craters is similar to that of the flattening of the red spectra by solar-proton irradiation, if the cut-off radius of the size distribution is $1 \mathrm{~mm}$ and its slope is -3 -for the whole size-range. If the contribution of ejecta is taken into account, a cut-off size of $1 m$, with the same slope, renders a collisional resurfacing-timescale in the required range.

The model presented here can be seen as an exercise to illustrate a scenario of collisional and radiation balance to understand the distribution of spectral slopes in the Trojan swarms. Naturally, refinements are needed to represent better reality. For example, our estimates of the collisional resurfacing timescales bear large uncertainties, mainly due to our lack of knowledge of the size distribution of small particles around the Lagrangian points of the orbit of Jupiter. On the other hand, the information regarding visual spectral slopes of known dynamical-family members (Fornasier et al. 2007), indicates that most dynamical families are remarkable uniform. With the noticeable exception of the one of 
Eurybathes, they are mostly moderately red, suggesting a correlation with age. Eventually, the calculations presented here could be used to understand such a potential trend.

Finally, a comment on how the scenario would change if the mass-loss rate of water ice at $120^{\circ} \mathrm{K}$, based in realistic rotation rates and albedos where to be higher than estimated here. In this case mass loss due to sublimation would be higher and the exposed surface ice could have been lost from the Trojans in their current orbit. A different orbit is not required.

\section{Acknowledgements}

We are grateful for the comments of an anonymous referee, which have helped to improve this article greatly, particularly regarding the sublimation rate of water ice.

GS has been supported by Italian Space Agency contract n. I/015/07/0 (Studi di Esplorazione Sistema Solare). A.B-N acknowledges partial support by the US-Israel Binational Science foundation. MDM acknowledges partial support by UBACyT X465.

\section{References}

Bar-Nun,A., Dror, J., Kochavi,E., Laufer, D. 1987. Amorphous water ice and its ability to trap gases. Physical Review B, 35, 2427-2435.

Bar-Nun, A., Pat-El, I., Laufer, D. 2007. Comparison between the findings of Deep Impact and our experimental results on large samples of gas-laden amorphous ice. Icarus, $\mathbf{1 9 1 , ~ 2 , ~ 5 6 2 - 5 6 6 . ~}$

Beauge, C., Roig, F. 2001. A Semi-analytical Model for the Motion of the Trojan 
Asteroids: Proper Elements and Families. Icarus, 153, 2, 391-415.

Bendoya.,P, Cellino, A, Di Martino, M., Saba, L. 2004. Spectroscopic observations of Jupiter Trojans. Icarus, 168, 2, 374-384.

Binzel, R.P. and Sauter, L.M. 1992. Trojan, Hilda, and Cybele asteroids - New lightcurve observations and analysis. Icarus, 95, 222-238.

Brunetto, R., Roush, T.L. 2008. Impact of irradiated methane ice crusts on compositional interpretations of TNOs. Astronomy and Astrophysics, 481, 879-882.

CRC-Handbook of Chemistry and Physics, 1981-1982, The Chemical Rubber Co.Cleveland $\mathrm{OH}$.

Cruikshank, D. P., Dalle Ore, C. M., Roush, T. L., Geballe, T. R., Owen, T. C., de Bergh, C., Cash, M. D.; Hartmann, W. K. 2001. Constraints on the Composition of Trojan Asteroid 624 Hektor. Icarus, 153, Issue 2, 348-360.

Dell'Oro, A., Marzari, P., Paolicchi F., Dotto, E., Vanzani, V. 1998. Trojan collision probability: a statistical approach. Astronomy and Astrophysics, 339, 272-277.

Emery, J. P. , Brown, R. H. 2003. Constraints on the surface composition of Trojan asteroids from near-infrared (0.8-4.0 $\mu \mathrm{m})$ spectroscopy, Icarus, 164, 104-121.

Emery, J. P. , Brown, R. H. 2004. The surface composition of Trojan asteroids: constraints set by scattering theory. Icarus, 170, 131-152.

Emery J.P., Cruikshank D.P. , Van Cleve J. 2006. Thermal emission spectroscopy (5.2-38 $\mu \mathrm{m})$ of three Trojan asteroids with the Spitzer Space Telescope: Detection of fine-grained silicates. Icarus, 82 , 2, 496-512.

Fernández, Y. R. , Sheppard, S. S. , Jewitt, D. C. 2003. The Albedo Distribution of Jovian Trojan Asteroids. The Astronomical Journal, 126, 1563-1574. 
Fornasier, S., Dotto, E., Hainaut, O., Marzari, F., Boehnhardt, H., de Luise, F., Barucci, M. A. 2007. Visible spectroscopic and photometric survey of Jupiter Trojans: Final results on dynamical families. Icarus 190, 2, 622-642.

Gil-Hutton, R. 2002. Color diversity among Kuiper belt objects: The collisional resurfacing model revisited. Planetary and Space Science, 50, 1, 57-62.

Gosling, J.T., 2007, The Solar wind, in: Encyclopedia of the Solar System 2nd Edition, L.McFadden, P.R. Weissman, T.V. Johnson Eds, Academic Press, pp 99-116

Hartmann, W. K., Binzel, R.P., Tholen, D.J., Cruikshank, D. P., Goguen, J. 1988. Trojan and Hilda asteroid light-curves. I - Anomalously elongated shapes among Trojans (and Hildas?). Icarus, 73, 487-498.

Holsapple K.A. 1993. The scaling of impact processes in planetary sciences. Annu. Rev. Earth Planet. Sci., 21, 333-374.

Hudson R.L., Palumbo M.E., Strazzulla G., Moore M.H., Cooper J.F., , Sturner S.J., 2008, Laboratory studies of the chemistry of TNO surface materials, in: The Solar System beyond Neptune, The University of Arizona Space Science Series, M. A. Barucci, H. Boehnhardt, D. P. Cruikshank, A. Morbidelli (editors), University of Arizona Press, Tucson, pp 507-523

Jewitt, D.C. 2002, From Kuiper Belt Object to Cometary Nucleus: The Missing Ultrared Matter. The Astronomical Journal, 123, 2, 1039-1049.

Jewitt, David C., Luu, Jane X. 1990. CCD spectra of asteroids. II - The Trojans as spectral analogs of cometary nuclei. Astronomical Journal, 100, 933-944.

Jewitt, David C., Trujillo, Chadwick A., Luu, Jane X. 2000. Population and Size Distribution of Small Jovian Trojan Asteroids. The Astronomical Journal, 120, 2, 
1140-1147.

Lanzerotti L. J., Brown, W. L., Poate, C. M., Augustyniak, W. M. 1978. Low energy cosmic ray erosion of ice grains in interplanetary and interstellar media. Nature $\mathbf{2 7 2}$, 431-433.

Marzari, F., Farinella, P., Davis, D. R., Scholl, H., Campo Bagatin, A. 1997. Icarus, 125, 1, 39-49.

Lisse, C. M., VanCleve, J., Adams, A. C., A’Hearn, M. F., Fernndez, Y. R., Farnham, T. L., Armus, L., Grillmair, C. J., Ingalls, J., Belton, M. J. S., Groussin, O., McFadden, L. A., Meech, K. J., Schultz, P. H., Clark, B. C., Feaga, L. M., Sunshine, J. M. 2006. Spitzer Spectral Observations of the Deep Impact Ejecta. Science, 313, 5787, 635-640.

Mayer,E., and Pelzer,R., 1986. Astrophysical implications of amorphous ice - A microporous solid. Nature, 319, 298-301.

Melita, M.D., Licandro, J., Jones, D., Wiliams, I.P. 2008a. Physical properties and orbital stability of the Trojan asteroids. Icarus, 195, 2, 686-697.

Melita, M.D., Duffard, R., Williams I.P., Jones, D.C., Licandro, J., Ortiz J.L. $2008 b$. Lightcurves of 21 Trojan Asteroids. $A \& \mathcal{E} A$, Submitted.

Moroz, L. V., Baratta, G., Strazzulla,G., Starukhina,L. V., Dotto, E., Barucci, . M.A., Arnold, G., Distefano, E., 2004. Optical alteration of complex organics induced by ion irradiation: I. Laboratory experiments suggest unusual space weathering trend. Icarus 170, $214-228$

Notesco, G., Bar-Nun, A., Owen, T. 2003. Gas trapping in water ice at very low deposition rates and implications for comets. Icarus, 162, 1, 183-189.

O'Brien, D.P., Greenberg, R., Richardson, J.E. 2006. Craters on asteroids: Reconciling 
diverse impact records with a common impacting population. Icarus, $\mathbf{1 8 3}, \mathbf{1 ,}$ 79-92.

Petit, J.M. , Farinella, P. 1993. Modelling the outcomes of high-velocity impacts between small solar system bodies. Celestial Mechanics and Dynamical Astronomy, 57, 1-2, 1-28.

Richardson, J.E., Melosh, H.J., Lisse, C.M., Carcich, B. 2007. A ballistics analysis of the Deep Impact ejecta plume: Determining Comet Tempel 1's gravity, mass, and density. Icarus, 190, 2, 357-390.

Sack, N.J. and Baragiola, R.A., 1993. Sublimation of vapor-deposited water ice below 170 K, and its dependence on growth conditions. Phys. Rev. B, 48, 99739978.

Yang, B, Jewitt, D. 2007. Spectroscopic Search for Water Ice on Jovian Trojan Asteroids. The Astronomical Journal, 134, 1, 223-228.

Wetherill, G. W. 1967. Collisions in the asteroid belt. J. Geophys. Res., 72, 2429-2444.

Ziegler, J. F., Biersack, J. P., and Littmark, U., 1996. The Stopping and Range of Ions in Solids, Pergamon Press, New York. 


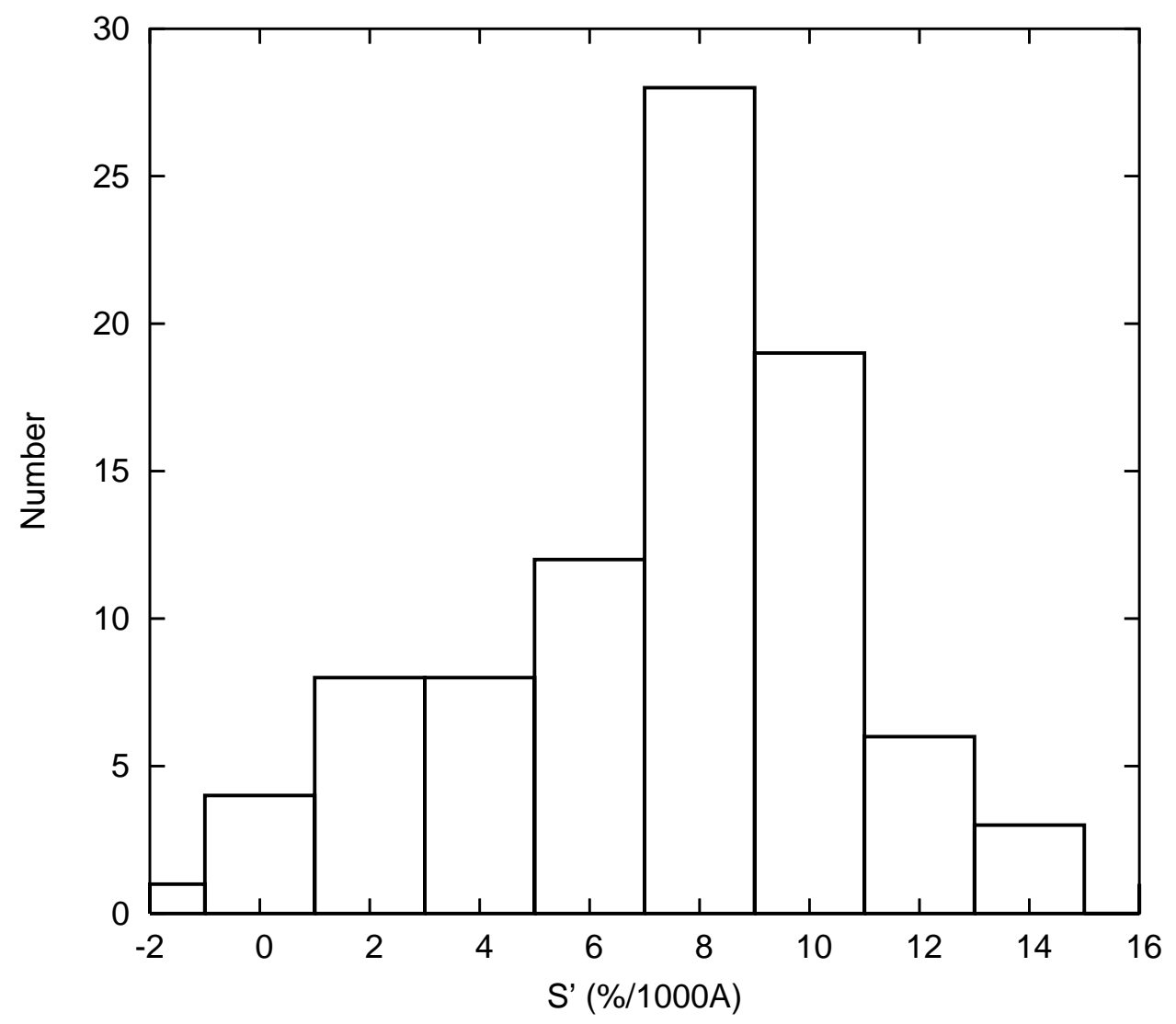

Fig. 1. - Histogram of the slopes of visual reflection spectra normalized at $6000 \AA$ for the Trojan asteroids. The sources of this data can be found in Melita et al. 2008. 


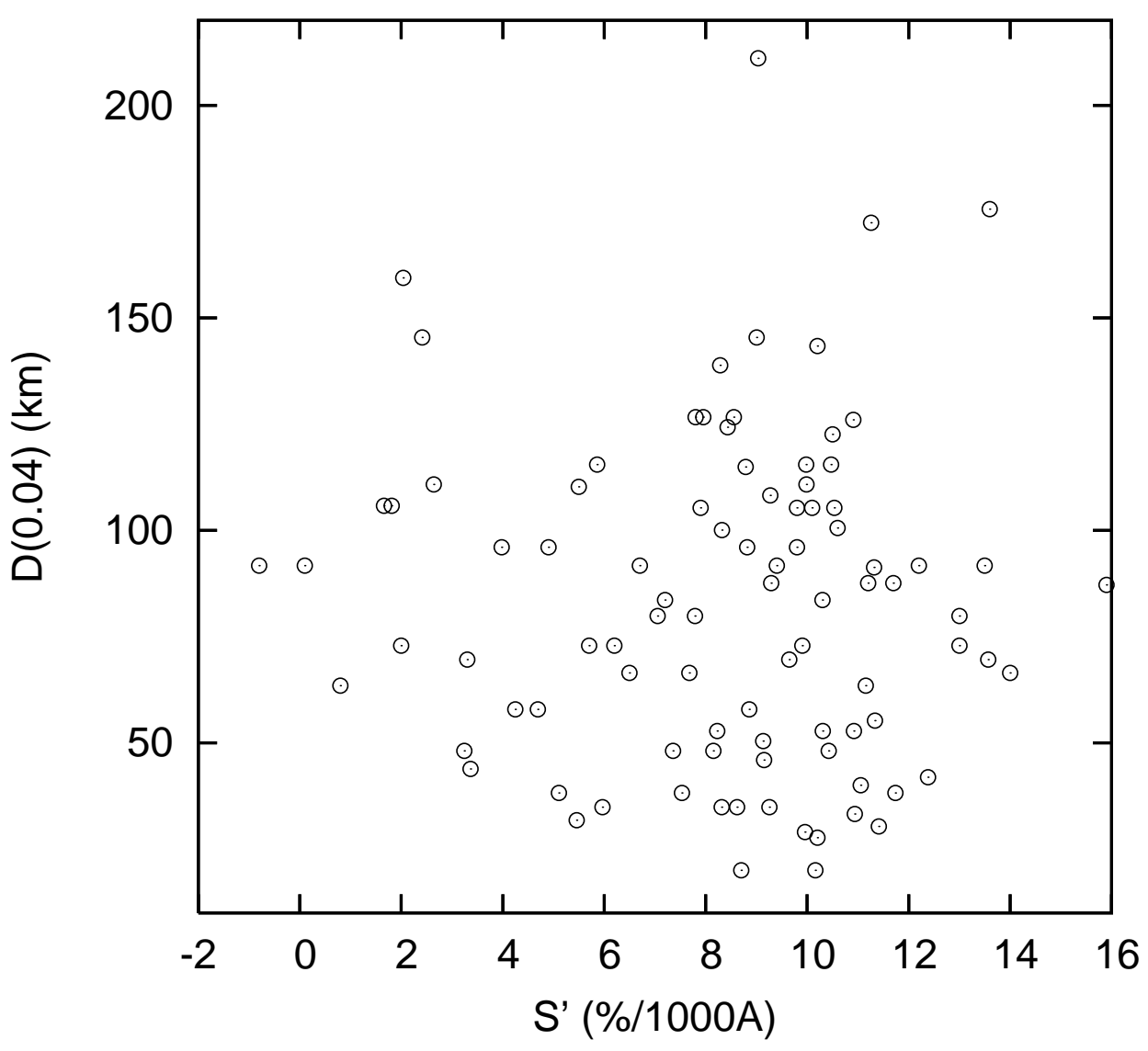

Fig. 2.- Slopes of visual reflection spectra normalized at $6000 \AA$ vs. $\mathrm{D}(0.04)$, the physical diameter, assuming an albedo of 0.04 , for the Trojan asteroids. Data taken form Melita et al. 2008. 

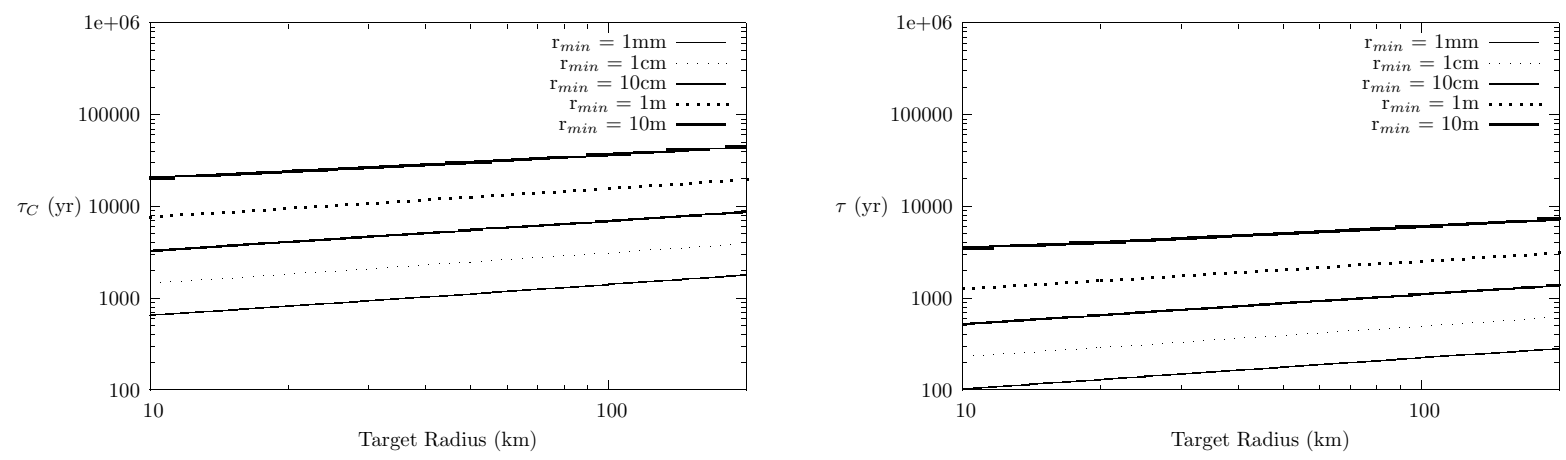

Fig. 3.- Left: Timescale in which the whole body is covered with craters, $\tau_{C}$, as a function of the radius of the target, for the size range of the known Trojan asteroids. Right: Timescale of collisional resurfacing, assuming a value of $l=25, \tau$, as a function of the radius of the target, for the size range of the known Trojan asteroids. See text for details. 